\title{
Rule Based Segmentation and Subject Identification Using Fiducial Features and Subspace Projection Methods
}

Article in Journal of Computers · June 2007

DOI: 10.4304/jcp.2.4.68-75 · Source: DOAJ

CITATIONS

2

2 authors, including:

Erhan A. Ince

Eastern Mediterranean University

34 PUBLICATIONS 70 CITATIONS

SEE PROFILE
READS

34

Some of the authors of this publication are also working on these related projects: 


\title{
Rule Based Segmentation and Subject Identification Using Fiducial Features and Subspace Projection Methods
}

\author{
Erhan AliRiza İnce \\ Department of Electrical and Electronic Engineering, Eastern Mediterranean University, North Cyprus \\ Email: erhan.ince@emu.edu.tr \\ Syed Amjad Ali \\ Department of Computer Technology and Information Systems, Bilkent University, Turkey \\ Email: syedali@bilkent.edu.tr
}

\begin{abstract}
This paper describes a framework for carrying out face recognition on a subset of standard color FERET database using two different subspace projection methods, namely PCA and Fisherfaces. At first, a rule based skin region segmentation algorithm is discussed and then details about eye localization and geometric normalization are given. The work achieves scale and rotation invariance by fixing the inter ocular distance to a selected value and by setting the direction of the eye-to-eye axis. Furthermore, the work also tries to avoid the small sample space (S3) problem by increasing the number of shots per subject through the use of one duplicate set per subject. Finally, performance analysis for the normalized global faces, the individual extracted features and for a multiple component combination are provided using a nearest neighbour classifier with Euclidean and/or Cosine distance metrics.
\end{abstract}

Index Terms-Skin color segmentation, color FERET database, geometric normalization, feature extraction, subspace analysis methods.

\section{INTRODUCTION}

Extracting face regions containing fiducial points and recovering pose are two challenging problems in computer vision. Many practical applications such as video telephony, hybrid access control, feature tracking and model based low bit rate video transmission require feature extraction and pose recovery.

There exist various methods for the detection of facial features and a detailed literature survey about these techniques is available in [1]- [6]. One of the very first operations that is needed for facial feature detection is the face localization. To achieve face localization many approaches such as segmentation based on skin color [2], [3], clustering [7], Principal Component Analysis [8], and

This paper is based on "An Adept Segmentation Algorithm and its Application to Extraction of Local Regions Containing Fiducial Points," by E. A. Ince and S. A. Ali, which appeared in the Proceedings of the $21^{\text {st }}$ International Symposium on Computer and Information Sciences, ISCIS 2006, Istanbul, Turkey, November 1-3, 2006, (C)Springer-Verlag Berlin Heidelberg 2006.

This work was supported by the Research Advisory Board of Eastern Mediterranean University under Seed Money Project EN-05-02-01. neural nets [9] have been proposed. Once the face is localized, facial features can then be extracted from the segmented face region by making use of image intensity, chromaticity values and geometrical shape properties of the face.

When using appearance based methods [10], [11] an image of size $(n \times m)$ pixels is represented in an $(n \times m)$ dimensional space. In practice, this many spaces are too large to allow robust and fast face recognition. A common way to attempt to resolve this problem is to use dimensionality reduction techniques [8], [12], [13], [14].

Recently, component based classifiers have shown promissing results in face recognition tasks [15], [16] and [17]. Results in [16] are based on images from a nonstandard database and [17] uses images acquired by an automated surveillance system and also synthetic images generated by 3D models. Motivated by the absence of a component-based analysis for a standard image database such as the color FERET database, this paper utilizes the adept segmentation algorithm suggested in [18] to localize the eye centers, normalizes the global images and extracts the facial features to classify each independently. These results are then compared with those obtained from normalized versions of holistic face images. Finally, recognition rate for a multiple component classification is obtained and compared with previous results.

The paper is organized as follows: section II talks about the database used which is a subset of the standard color FERET database and explains the expansion of the database by using one duplicate set per person so that the number of images per subject would be eight instead of four, which is the case in most publications. Section III discusses some existing skin region discrimination techniques and also provides details about a new rule based skin region segmentation algorithm. Additionally, feature map generation which is required for eye localization is also covered in this section. Section IV discusses the general structure of the face recognition system and provides details about geometric normalization. Section 
V explains two sub-space analysis methods: the Principal Component Analysis and the Fisher-faces methods. Section VI analyzes recognition performance for normalized global images, the individual extracted features and a multiple component classification. The analysis is based on a nearest-neighbour classifier using Euclidean and/or Cosine distance metrics. Lastly, section VII summarizes the findings of the work.

\section{The Color FERET DATABASE}

The database used in the simulations is a subset of the color FERET database [19] , [20] which has been created with an access system application in mind. The database is composed of 256 images of 32 different subjects chosen randomly with eight shots per subject. Half of these pictures come from a duplicate set which has been taken at a different time under different illumination conditions. The idea of duplicate set is based on the fact that people trying to get access for a specific research lab will turn up at different times wearing different clothes plus their hair may be done differently. Also considering that an authorized person will not pose in front of the acquisition camera at an angle more than 22.5 degrees only the FA, FB, QL, QR, RB and RC poses have been considered and profile left and right images have been intentionally excluded. FA and FB are frontal images, QR and QL are poses with the head turned about 22.5 degrees left and right respectively, and $\mathrm{RB}$ and $\mathrm{RC}$ are random images with the head turned about 15 degrees in either direction. This study uses only the smaller size images that are $(256 \times 384)$.

\section{SEgMENTATION}

The segmentation of skin regions in color images is a preliminary step in several face recognition algorithms. Many different methods for discriminating between skin and non-skin pixels are available in the literature. Details about some of these and a new rule based segmentation algorithm is provided in what follows. The section also describes the steps of feature map generation which can be used for eye localization.

\section{A. Skin Region Segmentation}

In the literature various skin color predicates have been used to extract the region corresponding to the skin area of the human face. One approach as described by [21] and [22] is to compare the input image with a Gaussian distribution model which can be obtained by supervised training. The images used for the generation of the color model must be obtained from people of different races, ages and gender under varying illumination conditions. In [21], it was noted that human skin colors differ more in brightness than in colors. Hence it was suggested that a normalized $R G B$ model is considered. This model expresses the colors of each pixel as a combination of $R, G, B$ components and the brightness by $I=R+G+B$.
To avoid being sensitive to brightness value of the pixels, each color component is normalized with $I$ as:

$$
r=\frac{R}{I} \quad g=\frac{G}{I} \quad b=\frac{B}{I}
$$

Empirical results indicate that the color distribution in normalized $R G B$ space can be expressed by a $2 D$ Gaussian distribution. Other approaches as suggested by [23], are to use the standard HSV hue definition and the brightness and gamma invariant (BGI) hue introduced by Finlayson and Schaefer:

$$
\begin{gathered}
H_{s t d}=\arccos \left(\frac{0.5(r-g)+0.5(r-b)}{\sqrt{(r-g)^{2}+(r-b)(g-b)}}\right) \\
H_{b g i}=\arctan \left(\frac{\log (r)-\log (g)}{\log (r)+\log (g)-2 \log (b)}\right)
\end{gathered}
$$

This work uses both the $R G B$ and the normalized $r$ - $g$ color spaces for skin segmentation as suggested by [18]. The upper and lower thresholds for the skin region is obtained by using the polynomial model suggested by [24]. A raw binary mask $B M$ is generated using the three rules listed below and then refined by selecting the largest connected component in the region and then filling the holes inside this selected region.

$$
\begin{aligned}
& \text { S1. } f_{\text {lower }}(r)<g<f_{\text {upper }}(r) \\
& \text { S2. } R>G>B \\
& \text { S3. } R-B>10 \\
& B M= \begin{cases}1 & \text { if all rules } \mathrm{S} 1, \mathrm{~S} 2 \text { and } \mathrm{S} 3 \text { are true, } \\
0 & \text { otherwise. }\end{cases}
\end{aligned}
$$

The final binary mask can be obtained by closing the gaps (holes) connected to the background in the upper part of the binary image which are mainly caused by eyes and eyebrows in a left or right rotated head. Fig. 1 depicts the process of skin segmentation on a quarter left subject from the color database.
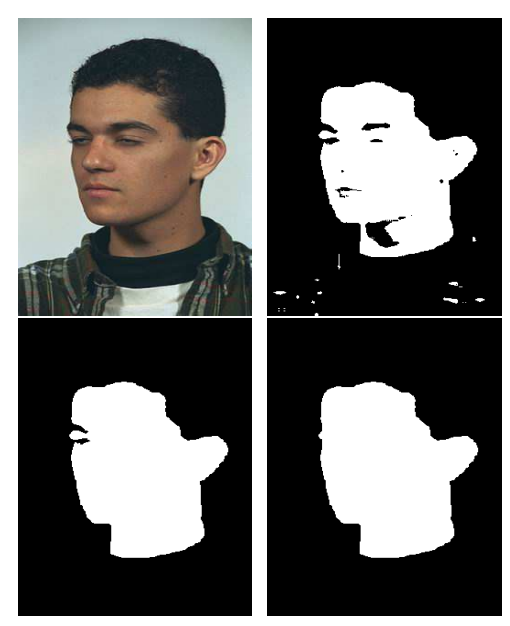

Figure 1. Left to right: (a) Original image (b) Binary mask $(B M)(\mathrm{c})$ Largest connected binary mask with holes filled (d) Binary mask after closing the gaps. 


\section{B. Feature Map Generation for Eye Localization}

Most approaches for eye and mouth detection are template based [15], [25]. However, in this paper we directly locate eyes based on measurements derived from the $r-g, R G B$ and $Y C_{b} C_{r}$ color space components of the images. In order to locate the possible eye region we first create a feature map $(F M)$ based on conditions $S 1-S 4$.

$$
\begin{aligned}
& f_{\text {upper }}(r)=-1.3067 r^{2}+1.0743 r+0.1452 \\
& f_{\text {lower }}(r)=-0.7760 r^{2}+0.5601 r+0.1766
\end{aligned}
$$

S1. $f_{\text {lower }}(r)<g<f_{\text {upper }}(r)$

S2. $R>G>B$

S3. $R-G>10$

S4. $65<C_{b}<125$ and $135<C_{r}<165$

$$
F M= \begin{cases}1 & \text { if } \mathrm{S} 1, \mathrm{~S} 2, \mathrm{~S} 3 \text { and } \mathrm{S} 4 \text { are true } \\ 0 & \text { otherwise. }\end{cases}
$$

Once $F M$ is obtained it is complemented and the components touching the borders are cleared to obtain the composite feature map $(C F M)$. Afterwards, the $C F M$ is masked by the binary face mask $(B M)$ obtained in previous section and finally the eye region is extracted by cropping part of the global image as dictated by (6):

$$
\begin{aligned}
F M_{\text {eye }}= & C F M(\text { toprow }+0.19 * \text { hindex }: \text { toprow } \\
& +(0.52 * \text { hindex }))
\end{aligned}
$$

where toprow and hindex are as defined in [18]. Fig. 2 depicts the resultant images after various steps of the algorithm.

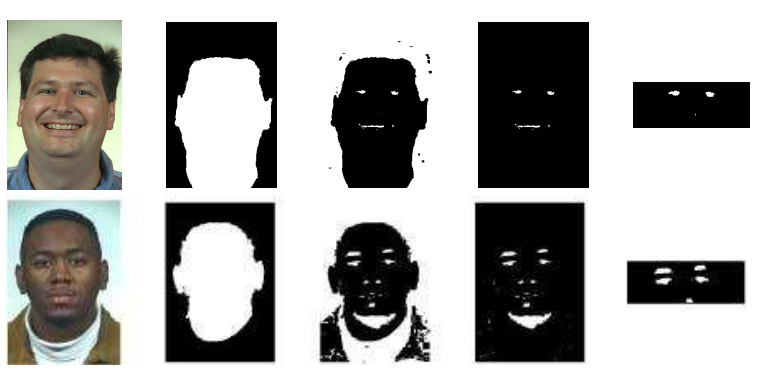

Figure 2. Feature map generation for eyes.

Following the verification rules for eyes as defined in [18], localization can be completed as shown by Fig. 3 .
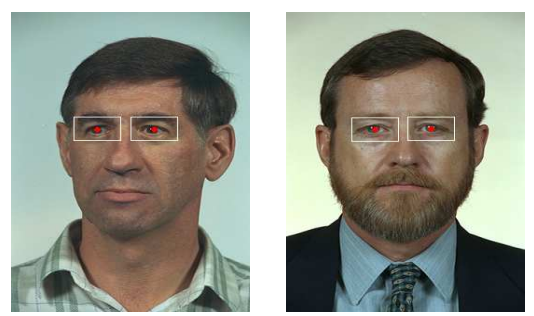

Figure 3. Localized Eyes.

\section{Face Recognition System}

An illustration of the general subspace face recognition system in the form of a block diagram is depicted in Fig. 4. A mean subtracted normalized probe image is projected into the subspace and then its projection is compared to stored projections of gallery images into the same subspace. The system then computes a set of distances between the probe and the gallery projection coefficients. A match corresponds to the smallest distance among all sets. It is worth noting here that normalization is carried out only when the input image is a head and shoulders image. For individual feature regions this step is avoided mainly because feature extraction is carried out after geometric normalization in our work.

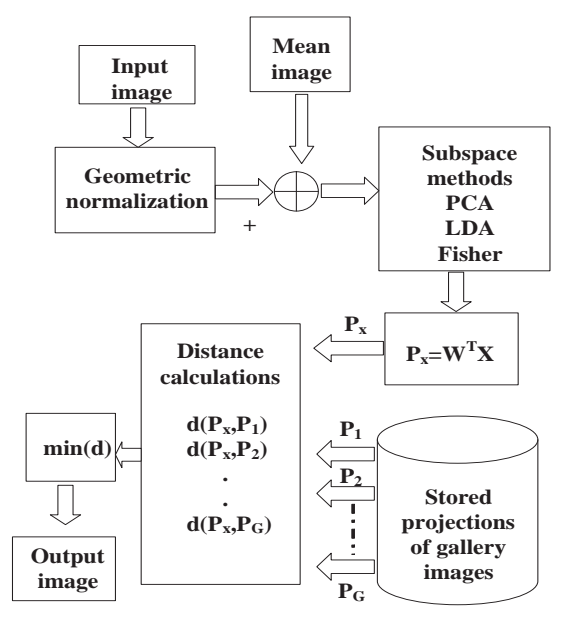

Figure 4. General subspace face recognition system.

\section{A. Geometric Normalization}

In order to perform face normalization, one needs to detect the coordinates of the eye centers and the midpoint between them. Using the left and right eye coordinates, the angle and the sense of rotation can be determined for de-rotating the faces in order to make the line connecting the eye centers horizontal. If we denote the midpoint before rotation as $\left(x_{m b}, y_{m b}\right)$ the new location of this point after the rotation, $\left(x_{m a}, y_{m a}\right)$, can be obtained using the formulas below

$\left[\begin{array}{l}x_{m a} \\ y_{m a}\end{array}\right]=\left[\begin{array}{cc}\cos \theta & \sin \theta \\ -\sin \theta & \cos \theta\end{array}\right]\left[\begin{array}{l}x_{m b}-x_{c} \\ y_{m b}-y_{c}\end{array}\right]+\left[\begin{array}{l}x_{c} \\ y_{c}\end{array}\right]$

It must be noted that MATLAB chooses to rotate images taking the center of the image, $\left(x_{c}, y_{c}\right)$, as a reference point. Once the angle is corrected a scale factor must be computed for scaling up or down the rotated image to let the inter eye distance be equal to a previously decided constant value. If we denote the midpoint coordinates on the angle corrected image as $\left(x_{m a}, y_{m a}\right)$ then the new location after the scaling, $\left(x_{a s}, y_{a s}\right)$, can be obtained as

$$
\left[\begin{array}{l}
x_{a s} \\
y_{a s}
\end{array}\right]=\left[\begin{array}{cc}
s f & 0 \\
0 & s f
\end{array}\right]\left[\begin{array}{l}
x_{m a} \\
y_{m a}
\end{array}\right]
$$


Finally, the face images need to be cropped taking this new mid point as a reference. The width and height of the region to be cropped out can be based on some multiple of the inter eye distance. An example showing the steps described is depicted in Fig. 5. For rotation and resizing operations bi-cubic interpolation was used.

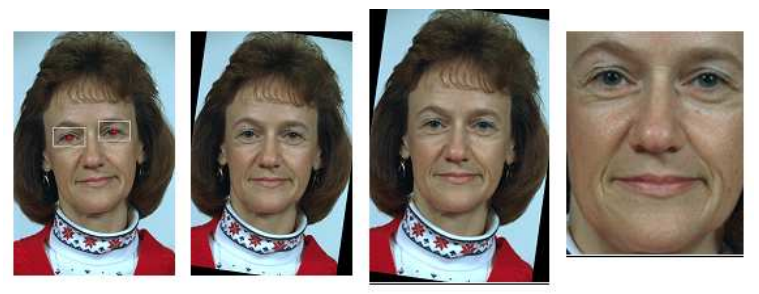

Figure 5. Geometric Normalization of Head and Shoulder Images.

Fig. 6 shows the normalized faces and extracted individual feature components for two Asians and two opposite sex Caucasians.

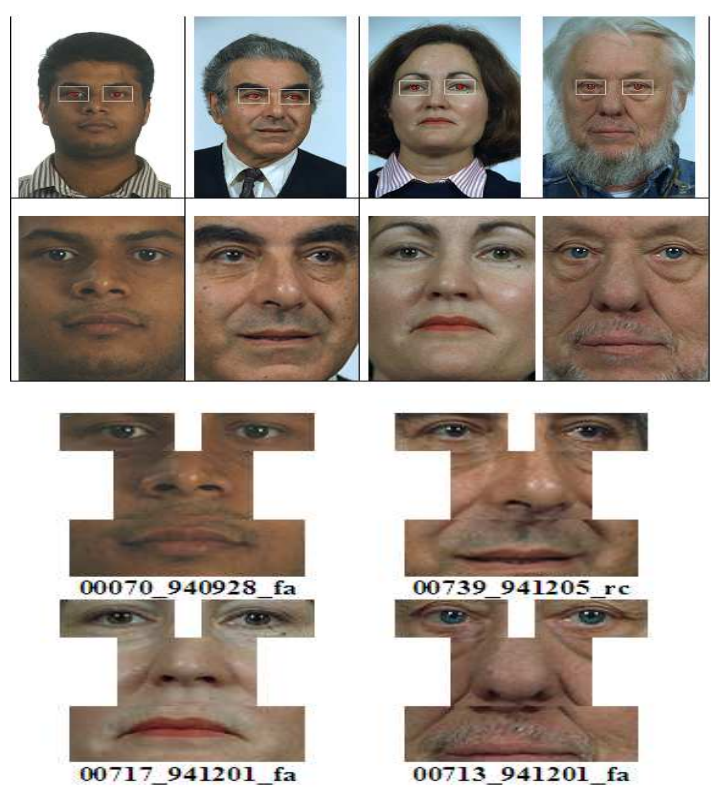

Figure 6. (a)Normalization of Head and Shoulder Images (b) Extracted Feature regions for different subjects.

\section{Subspace Analysis Methods}

When using holistic approach for face recognition, an image of size $(n \times m)$ is represented in an $(n \times m)$ dimensional space. In practice this many spaces is too large to allow robust and fast face recognition. A common way to attempt to resolve this problem is to use dimensionality reduction techniques. What follows, provide details about two such subspace techniques.

\section{A. Principal Component Analysis (PCA)}

As correlation based methods are computationally expensive and require fairly large amounts of storage researchers have naturally explored dimensionality reduc- tion schemes. One commonly used dimensionality reduction scheme is the Principal Component Analysis (PCA) also known as the Kaehunen-Loeve transform.

For a set of $N$ sample images $\left\{I_{1}, I_{2}, \ldots, I_{N}\right\}$ taking values in an $n$-dimensional image space, assume that each image belongs to one of $C$ classes $\left\{X_{1}, X_{2}, \ldots ., X_{C}\right\}$. In addition let $W$ represent a linear transformation that maps the $n$-dimensional original space onto a $m$-dimensional reduced space. The new feature vectors $\mathbf{y}_{k} \in \Re^{m}$ can be defined as

$$
\mathbf{y}_{k}=W^{T} \mathbf{x}_{k} \quad k=1,2, \ldots, N
$$

the transformation matrix $W \in \Re^{n \times m}$ and has columns which are orthonormal. The total scatter matrix $S_{T}$ is defined as

$$
S_{T}=\sum_{k=1}^{N}\left(\mathbf{x}_{k}-\mu\right)\left(\mathbf{x}_{k}-\mu\right)^{T}
$$

where $N$ represents the number of sample images and $\mu$ is the mean image of all samples. The scatter of the transformed feature vectors is $W^{T} S_{T} W$. The projection $W_{\text {opt }}$ is chosen to maximize the determinant of the total scatter matrix of the projected samples

$$
W_{o p t}=\arg \max _{W}\left|W^{T} S_{T} W\right|=\left[\mathbf{w}_{1}, \mathbf{w}_{2} \cdots \mathbf{w}_{m}\right]
$$

where $\mathbf{w}_{i}$ are the set of $n$-dimensional eigenvectors of $S_{T}$ corresponding to the $m$ largest eigenvalues. Since these eigenvectors have the same dimension as the original images, they are referred to as Eigenfaces [26].

\section{B. Fisherface $(P C A+L D A)$ Analysis}

While the eigen approach allows for a low dimensional representation of the training images, it implicitly reduces the discrimination between training images. While this is a useful property for compactly coding images, it may have some undesirable consequences when it comes to classification.

The Fisherface method expands the training set to contain multiple images of each person, providing examples of how a person's face may change from one image to another due to variations in lighting conditions, facial expressions etc. The training set is defined with $K$ classes with $N_{j}$ samples per class. The mean image of class $C_{j}$ is $u_{j}$ and the mean image of all samples is $u$.

$$
u_{j}=\left(1 / N_{j}\right) \cdot \sum_{x \in C_{j}} x \quad \text { and } \quad u=(1 / N) \cdot \sum_{j=1}^{K} \sum_{x \in C_{j}} x
$$

The algorithm then computes the three scatter matrices, representing the within-class $\left(S_{W}\right)$, between-class $\left(S_{B}\right)$ 
and the total scatter $\left(S_{T}\right)$ matrices as:

$$
\begin{aligned}
S_{W} & =\frac{1}{N} \sum_{j=1}^{K} \sum_{x \in C_{j}}\left(x-u_{j}\right)\left(x-u_{j}\right)^{T} \\
S_{B} & =\frac{1}{N} \sum_{j=1}^{K} N_{j}\left(u_{j}-u\right)\left(u_{j}-u\right)^{T} \\
S_{T} & =\frac{1}{N} \sum_{j=1}^{K} \sum_{x \in C_{j}}(x-u)(x-u)^{T} .
\end{aligned}
$$

If $S_{W}$ is not singular, LDA which is also known as Fisher Linear Discriminant (FLD) tries to find a projection $W_{o p t}=\left(w_{1}, w_{2}, \ldots, w_{L}\right)$ that satisfies the Fisher criterion:

$$
W_{o p t}=\arg \max _{W}\left|\frac{W^{T} S_{B} W}{W^{T} S_{W} W}\right| .
$$

where $W_{\text {opt }}=\left(w_{1}, w_{2}, \ldots, w_{L}\right)$ are the eigenvectors of $S_{W}^{-1} S_{B}$ corresponding to $L$ largest eigenvalues $\left(\lambda_{1}, \lambda_{2}, \ldots, \lambda_{L}\right)$.

However if $S_{W}$ is singular then the inverse of $S_{W}$ does not exist. To alleviate this problem, the Fisherface method is generally adopted. Fisherface method makes use of PCA to project the image set to a lower dimensional space so that within-class scatter matrix $\widehat{S}_{W}$ is nonsingular and then applies the standard LDA.

The optimal projection $W_{\text {opt }}$ of Fisherface method is normally given as

$$
\begin{aligned}
& W_{o p t}^{T}=W_{f l d}^{T} W_{p c a}^{T} \\
& W_{P C A}=W_{o p t}=\arg \max _{W}\left|W^{T} S_{T} W\right| \\
& W_{P C A}=\left(w_{p c a 1}, \ldots, w_{p c a p}\right) \\
& W_{f l d}=\arg \max _{W}\left|\frac{W^{T} \widehat{S}_{B} W}{W^{T} \widehat{S}_{W} W}\right| .
\end{aligned}
$$

where

$\widehat{S}_{W}=W_{P C A}^{T} S_{W} W_{P C A} \quad$ and $\quad \widehat{S}_{B}=W_{P C A}^{T} S_{B} W_{P C A}$

$W_{P C A}$ and $\left\{\lambda_{P C A i}\right\}_{i=1}^{p}$ are the eigenvectors and eigenvalues of $S_{T}$ respectively.

The use of PCA for dimension reduction in the Fisherface method has two purposes. First, when small sample size $(S 3)$ problem occurs $S_{W}$ becomes singular. PCA is used to reduce the dimension such that $S_{W}$ becomes nonsingular. The second purpose is that even though when there is no $S 3$ problem it helps reduce the computational complexity.

\section{Nearest Neighbour Classifier with Various Metrics}

In the testing phase, PCA or the Fisherfaces algorithm reads the subspace basis vectors generated during training. It then projects the probe (testing) images into the same sub-space and distance between all pairs of projected gallery (training) and probe images are computed. Both subspace algorithms discussed above were tested using the Euclidean and Cosine distance metrics as provided below

\section{C.1.0 Squared Euclidean Distance}

$$
d_{E D}(x, y)=\|x-y\|^{2}=\sum_{i=1}^{k}\left(x_{i}-y_{i}\right)^{2}
$$

\section{C.2.0 Cosine Distance}

$$
d_{C D}(x, y)=1-\frac{x \cdot y}{\|x\|\|y\|}=1-\frac{\sum_{i=1}^{k} x_{i} y_{i}}{\sqrt{\sum_{i=1}^{k}\left(x_{i}\right)^{2} \sum_{i=1}^{k}\left(y_{i}\right)^{2}}}
$$

\section{EXPERIMENTAL RESULTS}

The experimental results presented in this section are obtained using the subset of the color FERET database. The images used are frontal, quarter left, quarter right and faces with the head turned around 15 degrees in either direction. Profile images have intentionally been left out since for them only one eye can be extracted and this will prohibit the geometric normalization step. Without the normalization it is not possible to assume scale and rotation invariance.

Also for the first time, while using the color FERET database this work tries to increase the number of shots per subject (from 4 to 8 ) by using a duplicate set for each person. Even though, a mixture of images taken at different times and under different conditions may cause hardships for recognition this will allow more samples for training and in addition the small sample size problem that causes the within-class scatter matrix to become singular will be avoided.

Throughout the experiments three types of tests were administered. In the first category, the normalized head and shoulder images were used together with the two subspace analysis methods. Whereas in the second and third categories only individual feature images have been used as the gallery and probe images.

In what follows Table I summarizes the eye center localization and normalization results. It is worth noting that the normalization accuracy is a bit higher than the localization. This happens because even if the detected center coordinates are a reasonable number of pixels off from the actual center coordinates normalization can still be achieved.

TABLE I.

ACCURACY OF LOCALIZATION AND GEOMETRIC NORMALIZATION

\begin{tabular}{|lr|}
\hline Left eye & $98.05 \%$ \\
Right eye & $98.44 \%$ \\
\hline Correctly Normalized Images & $99.22 \%$ \\
Incorrectly Normalized Images & $0.78 \%$ \\
\hline
\end{tabular}

Tables II and III provide the face recognition rates when the subspace analysis method chosen is either PCA or Fisherfaces (PCA+LDA). In these experiments both the probe and the gallery images are picked from the 
normalized head and shoulder images of size $(141 \times 175)$. The results provided in the tables are for either $7: 1$ or $5: 3$ training to testing ratios. As stated by Kirby in [27], one expects that when the number of eigenvectors is roughly around $30 \%$ of the total number of images in the face database the PCA performance should start saturating. This is indeed the case here also. When the number of eigenvectors reaches 80 (256 images in total) the recognition rate (R.R.) saturates at 62.5 . The Fisherface method

TABLE II.

PRINCIPAL COMPONENT ANALYSIS FOR NORMALIZED IMAGES

\begin{tabular}{|c||c|c|}
\hline \multicolumn{1}{|c||}{ Eigenvectors } & \multicolumn{2}{c|}{ Recognition rate (R.R.) } \\
\cline { 2 - 3 } & $7: 1$ train/test & $5: 3$ train/test \\
\hline \hline 1 & $9.38 \%$ & $7.29 \%$ \\
10 & $37.5 \%$ & $41.46 \%$ \\
20 & $56.25 \%$ & $50.0 \%$ \\
40 & $59.38 \%$ & $59.38 \%$ \\
60 & $62.5 \%$ & $61.46 \%$ \\
80 & $62.5 \%$ & $61.46 \%$ \\
100 & $62.5 \%$ & $61.46 \%$ \\
150 & $62.5 \%$ & $61.46 \%$ \\
\hline
\end{tabular}

achieves relatively higher recognition rates in comparison to the PCA method (approximately 20\%). This is expected since the Fisherface method is an example of a class specific method and is able to discriminate well between classes even under severe variations in lighting and facial expressions. However, as depicted by Table III the performance gain achieved by using a different distance metric is not significant. This observation seems to agree with the findings of [28].

TABLE III.

FISHERFACES ANALYSIS FOR NORMALIZED IMAGES

\begin{tabular}{|c|c|c|}
\hline Features & R.R. with $d_{E D}(x, y)$ & R.R. with $d_{C D}(x, y)$ \\
\hline \hline 35 & $78.13 \%$ & $84.38 \%$ \\
45 & $81.25 \%$ & $81.25 \%$ \\
55 & $87.50 \%$ & $87.50 \%$ \\
75 & $81.25 \%$ & $84.38 \%$ \\
85 & $75.00 \%$ & $81.25 \%$ \\
95 & $65.63 \%$ & $68.75 \%$ \\
\hline
\end{tabular}

Tables IV and V give the R.R.s for PCA and Fisherface methods when applied to one individual feature at a time. Results obtained are similar to the ones stated in [29]. Experiments carried out with feature sets extracted from the normalized FERET database images indicate that no component alone is enough to attain a performance better than the one obtained when using the whole face. Parallel to the previous comparisons the Fisherface method again gives higher recognition rates than the PCA.

In this study, before using the extracted components for subspace analysis histogram equalization and contrast enhancement is applied to each extracted component. When the IMADJUST command of MATLAB is used $1 \%$ of the data is saturated at low and high intensities which would result in an increase in the contrast of the output image.

Finally, Table V shows that among individual classifications of all components, the left and right eyes result
TABLE IV.

PRINCIPAL COMPONENT ANALYSIS OF INDIVIDUAL COMPONENTS

\begin{tabular}{|c|c|c|c|c|}
\hline \multirow[t]{2}{*}{ Eigenvectors } & \multicolumn{4}{|c|}{ Recognition rate for $7: 1$ train/test ratio } \\
\hline & Right Eye & Left Eye & Nose & Mouth \\
\hline 1 & $13.67 \%$ & $8.59 \%$ & $4.69 \%$ & $9.77 \%$ \\
\hline 10 & $45.70 \%$ & $35.16 \%$ & $38.28 \%$ & $37.11 \%$ \\
\hline 20 & $55.86 \%$ & $45.70 \%$ & $42.58 \%$ & $42.97 \%$ \\
\hline 40 & $58.98 \%$ & $48.05 \%$ & $48.05 \%$ & $46.88 \%$ \\
\hline 60 & $58.98 \%$ & $48.05 \%$ & $48.05 \%$ & $49.61 \%$ \\
\hline 80 & $59.77 \%$ & $48.44 \%$ & $48.83 \%$ & $49.22 \%$ \\
\hline 100 & $60.16 \%$ & $49.22 \%$ & $48.44 \%$ & $49.22 \%$ \\
\hline \multirow[t]{3}{*}{150} & $60.94 \%$ & $50.00 \%$ & $48.83 \%$ & $49.22 \%$ \\
\hline & \multicolumn{4}{|c|}{ Recognition rate for 5:3 train/test ratio } \\
\hline & Right Eye & Left Eye & Nose & Mouth \\
\hline 1 & $10.03 \%$ & $6.51 \%$ & $4.69 \%$ & $7.94 \%$ \\
\hline 10 & $44.66 \%$ & $31.25 \%$ & $36.46 \%$ & $36.33 \%$ \\
\hline 20 & $51.95 \%$ & $37.37 \%$ & $41.80 \%$ & $41.41 \%$ \\
\hline 40 & $54.56 \%$ & $39.71 \%$ & $44.66 \%$ & $45.18 \%$ \\
\hline 60 & $55.60 \%$ & $41.02 \%$ & $45.96 \%$ & $46.48 \%$ \\
\hline 80 & $55.21 \%$ & $41.80 \%$ & $45.96 \%$ & $46.87 \%$ \\
\hline 100 & $55.47 \%$ & $41.54 \%$ & $45.96 \%$ & $46.74 \%$ \\
\hline 150 & $56.25 \%$ & $41.67 \%$ & $45.83 \%$ & $46.87 \%$ \\
\hline
\end{tabular}

TABLE V.

FISHERFACES ANALYSIS OF INDIVIDUAL COMPONENTS

\begin{tabular}{|c||c|c|c|}
\hline \multicolumn{1}{|c||}{ Component } & \multicolumn{3}{c|}{ Recognition Rate for PCA+LDA } \\
\cline { 2 - 4 } & Features & $d_{E D}(x, y)$ & $d_{C D}(x, y)$ \\
\hline \hline Right Eye & 40 & $75 \%$ & $75 \%$ \\
Left Eye & 40 & $62.5 \%$ & $75 \%$ \\
Nose & 40 & $65.63 \%$ & $68.75 \%$ \\
Mouth & 40 & $56.25 \%$ & $65.63 \%$ \\
\hline
\end{tabular}

in the highest two recognition rates.

Works presented in [17] and [29] state that multiple component based classifiers can achieve as good or better performance than the normalized whole face classifiers. In [25] and [29], it is also suggested that similarity scores of different features are integrated using one of the approaches below:

- choose the score of the most similar feature

- add the feature scores

- linear-combinate the feature scores giving each feature a different weight

Encouraged by these findings an extra set of simulations were run where the similarity scores for left eye (LE), right eye (RE) and mouth (M) components extracted from the subset of the color FERET database images were combined by adding them. The recognition rates attained by applying Principal Component Analysis with $7: 1$ and 5:3 training to testing ratios can be seen in Fig. 7. Clearly, the multiple component based classifier outperforms the whole face based one regardless of how many eigenvectors are used. For the 7:1 PCA, the performance gain is approximately $15 \%$ when the number of eigenvectors is above 60 , whereas for the $5: 3$ PCA this gain is approximately $10 \%$ for same number of eigenvectors.

\section{CONCLUSION}

The paper describes a framework for carrying out face recognition on a subset of the standard color FERET 


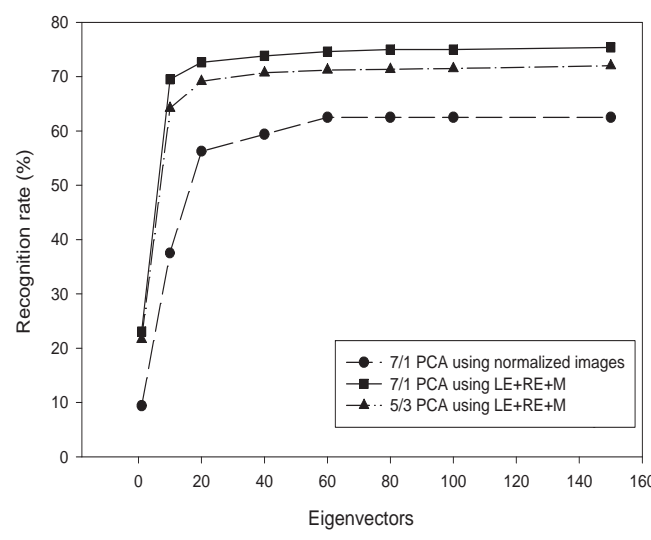

Figure 7. PCA recognition for multiple component versus whole face based classifiers.

database using two different subspace projection methods. Recognition performance for normalized global images, the individual extracted features and a multiple component classifier is provided. Analysis of the results indicate that when PCA is applied to either the normalized global images or the individual extracted components it would deliver approximately $20 \%$ lower recognition rate in comparison to the Fisherface method. This observation agrees with what other published works claim based on their experiments with various other image databases. Also, here it is once again confirmed that no single extracted component can deliver a higher recognition performance than the normalized global images. The de-rotation and re-scaling operations involved with geometric normalization results in a change in the pixel intensities due to interpolation. Therefore, in order not to compromise from recognition performance some post processing must be applied to each feature image. This work also shows that multiple features based classification will outperform the whole face based classifier. Use of reduced dimensionality analysis together with component-based classification not only brings higher performance but it also cuts down drastically on the computational costs. Finally, it is worth mentioning here that the recognition rates provided herein are very promising since the paper adopts a duplicate set for each person in order to increase the number of shots per class. Using duplicate sets implies that half the pictures are taken at a different time, under different lighting and with different backgrounds.

\section{ACKNOWLEDGMENT}

The work presented herein is an outcome of research carried out under Seed Money Project EN-05-02-01, granted by the Research Advisory Board of Eastern Mediterranean University.

\section{REFERENCES}

[1] Rein-Lien H., Abdel-Mottaleb M. : Face detection in colour images, IEEE Trans. on Pattern Analysis and Machine Intelligence, Vol. 24, No. 5, pp. 696-706, May 2002.
[2] Ming-Hsuan Y., Kriegnam D. J., Ahuja N. : Detecting faces in images: a survey, IEEE Trans. on Pattern Analysis and Machine Intelligence, Vol. 24, No. 1, pp. 34-58, January 2002.

[3] Alattar A. M., Rajala S. A. : Facial Features Localization In Front View Head And Shoulders Images, IEEE International Conference on Acoustics, Speech, and Signal Processing, Vol. 6, pp. 3557-3560, March 1999.

[4] Rein-Lien H., Abdel-Mottaleb M., Jain A. K. : Face detection in color images, Tech. Report MSU-CSE-01-7, Michigan State University, March 2001.

[5] Ínce E. A., Kaymak S., Çelik T. : Yüzsel Öznitelik Sezimi Için Karma Bir Teknik, 13. IEEE Sinyal Ísleme ve Íletisim Uygulamaları Kurultayl, pp. 396-399, May 2005.

[6] Hu M., Worrall S., Sadka A. H., Kondoz A. M. : Face Feature Detection And Model Design For 2D Scalable ModelBased Video Coding, International Conference on Visual Information Engineering, pp. 125-128, July 2003.

[7] Sung K., Poggio T. : Example based Learning for Viewbased Human Face Detection, C.B.C.L. , Paper No: 112, MIT, 1994.

[8] Moghaddam B., Pentland A. : Face Recognition using View-Based and Modular Eigenspaces, IEEE Transactions on Pattern Analysis and Machine Intelligence, Vol. 20, No. 1, pp. 23-38, January 1998.

[9] Rowley H., Baluja S., Kanade T. : Neural Network Based Face Detection, IEEE Trans. on Pattern Analysis and Machine Intelligence, Vol. 24, No. 1, pp. 34-58, January 2002.

[10] Murase H., Nayar S. K. : Visual Learning and Recognition of 3D Objects from Appeaarence, Int. Journal of Computer Vision, Vol. 14, pp. 5-24, 1995.

[11] Turk M., Pentland A. P. : Face Recognition Using Eigenfaces, Int. Conf on Computer Vision and Pattern Recognition, 1991.

[12] Martinez A. M., Kak A. C. : PCA versus LDA, IEEE Transactions on Pattern Analysis and Machine Intelligence, Vol. 23, No. 2, pp. 228-233, 2001.

[13] Zhao W., Chellappa R., Khrishnaswamy A. : Discriminant analysis of principal components for face recognition, IEEE Int. Conf. on Automatic Face and Gesture Recognition, pp. 336-341, 14-16 April, 1998.

[14] Belhumeur P. N., Hespanha J. P., Kriegman D. J. : Eigenfaces vs. Fisherfaces: Recognition Using Class Specific Linear Projection, IEEE Trans. on Pattern Analysis and Machine Intelligence, Vol. 19, No. 7, pp. 711-720, July 1997.

[15] Li S. Z., Jain A. K. : Handbook of Face Recognition, Springer-Verlag, 2005.

[16] Campos T. E., Feris R. S., Cesar R. M. : Eigenfaces versus Eigeneyes: First Step Towards Performance Assesment of Representations for Face Recognition, Lecture Notes in Artificial Intelligence, Vol. 1793, pp. 197-206, April 2000.

[17] Weyrauch B., Heisele B., Huang J., Blanz V. : Componentbased Face Recognition with 3D Morphable Models, Computer Vision and Pattern Recognition Conf., pp. 85-90, June 2004.

[18] Ince E. A., Ali S. A. : An Adept Segmentation Algorithm and its Application to Extraction of Local Regions Containing Fudicial Points,21st International Symposium on Computer and Information Sciences, ISCIS 2006, pp. 553562, November 2006.

[19] Phillips P. J., Wechsler H., Huang J., Rauss P. : The FERET database and evaluation procedure for face recognition algorithms, Image and Vision Computing J., Vol. 16, No. 5, pp. 295-306, 1998.

[20] Phillips P. J., Moon H., Rizvi S. A., Rauss P. J. : The FERET Evaluation Methodology for Face Recognition Algorithms, IEEE Trans. Pattern Analysis and Machine Intelligence, Vol. 22, pp. 1090-1104, 2000.

[21] Wang Y., Yuan B. : A novel approach for human face 
detection from color images under complex background, Journal of Pattern Recognition, Vol. 34, pp. 1983-1992, 2001.

[22] Campos T. E., Feris R. S., Cesar R. M. : Detection and Tracking of Facial Features in Video Sequences, Lecture Notes in Artificial Intelligence, Vol. 1793, pp. 197-206, 2000.

[23] Heseltine T., Pears N., Austin J., Chen Z. : Face Recognition: A Comparison of Appearance-Based Approaches, 7th Digital Image Computing: Techniques and Applications, pp. 59-68, Sydney, December 2003.

[24] Chiang C-C., Tai W-K., Yang M-T., Huang Y-T., Huang C-J. : A novel method for detecting lips, eyes and faces in real time, Real-Time Imaging 9 , Vol. 9, pp. 277-287, 2003.

[25] Brunelli R., Poggio T. : Face Recognition: Features versus Templates, IEEE Trans. on Pattern Analysis and Machine Intelligence, Vol. 15, No. 10, pp. 1042-1052, October 1993.

[26] Wang J., Zhang C., Shum H-Y. : Face Image Resolution Versus Face Recognition Performance Based on Two Global Methods, Asia Conference on Computer Vision, January 2004.

[27] Kirby M., Sirovich L. : Application of Karhunen-Loeve Procedure For the Characterization of Human Faces, IEEE Trans. PAMI, Vol. 12, pp. 103-108, 1990.

[28] Delac K., Grgic M., Grgic S. : A Comparative Study of PCA, ICA, and LDA, Proc. of the 5th EURASIP Conference focused on Speech and Image Processing,Multimedia Communications and Services, pp. 99-106, July 2005.

[29] Ivanov Y., Heisele B., Serre T. : Using Component Features for Face Recognition, 6th IEEE Int. Conf. on Automatic Face and Gesture Recognition, pp. 421-426, May 2004.

Erhan AliRiza Ince was born in Nicosia, Cyprus. Currently he is a full time academic member of the Electrical and Electronic Engineering Department at Eastern Mediterranean University, Famagusta - TRNC. He has received the BS and MS degrees in Electrical and Electronics Engineering from Bucknell University, Lewisburg PA, in 1990 and 1992 respectively, and the PhD degree in Communications from University of Bradford-UK, in 1997.

Dr. Ince's research interests include indoor/mobile communications, channel and source coding, Turbo codes, performance bounds and exact solutions, image processing and modelling. He has published over 27 scholarly articles and has been an IEEE member for the past 20 years.

Syed Amjad Ali was born in Karachi, Pakistan. He is currently a full time academic member at the Department of Computer Technology and Information Systems, Bilkent University, Ankara, Turkey. He received his BS, MS and $\mathrm{PhD}$ degrees in Electrical and Electronic Engineering from Eastern Mediterranean University, Famagusta - TRNC in 1999, 2001 and 2007 respectively. His research interests include communication theory, simulation and analysis of iterative channel coding schemes and image processing. Dr. Ali has over 15 scholarly published articles and has been an active IEEE member for the last 5 years. 